04

\title{
Кинетическая теория пристеночного слоя при произвольных условиях в газоразрядной плазме
}

\author{
(C) О. Мурильо, ${ }^{1}$ А.С. Мустафраев, ${ }^{1}$ В.С. Сухомлинов ${ }^{2}$ \\ ${ }^{1}$ Санкт-Петербургский горный университет, \\ 199106 Санкт-Петербург, Россия \\ ${ }^{2}$ Санкт-Петербургский государственный университет, фризический фракультет, \\ 198504 Петергоф, Санкт-Петербург, Россия \\ e-mail: rectorat@spmi.ru; v_sukhomlinov@mail.ru
}

Поступило в Редакцию 12 февраля 2019 г.

В окончательной редакции 12 февраля 2019 г.

Принято к публикации 1 апреля 2019 г.

\begin{abstract}
При произвольном соотношении между радиусом Дебая и длиной пробега иона на основе кинетического подхода решена самосогласованная задача о структуре возмущенного пристеночного слоя в газоразрядной плазме постоянного тока вблизи плоской поверхности, находящейся под отрицательным потенциалом относительно плазмы. Решение получено без искусственного разделения этого слоя на квазинейтральный „предслой“ и пристеночный слой, где существенно нарушается квазинейтральность. Учтены реальная функция распределения ионов в невозмущенной плазме, зависимость сечения перезарядки от энергии иона и ненулевое электрическое поле в невозмущенной плазме. Показано, что при сохранении средней энергии электронов структура возмущенного пристеночного слоя слабо зависит от вида функции распределения электронов. Установлено, что даже в предположении, что средняя энергия электронов много выше таковой для ионов, величина средней энергии ионов в невозмущенной плазме существенно влияет как на структуру квазинейтрального предслоя, так и на структуру части пристеночного слоя, где квазинейтральность отсутствует. Расчеты параметров ионного потока и структуры возмущенного пристеночного слоя согласуются с известными экспериментальными данными других авторов, не имевшими ранее адекватного толкования.
\end{abstract}

Ключевые слова: критерий Бома, пристеночный слой, уравнение Больцмана, газоразрядная плазма.

DOI: $10.21883 /$ JTF.2019.09.48064.51-19

\section{Введение и постановка задачи}

Рассмотрим структуру пристеночного слоя газоразрядной плазмы в случае, когда стенка находится под отрицательным потенциалом относительно плазмы. Такие слои образуются, в частности у стенок, ограничивающих объем плазмы, вблизи электрических зондов, около катода. Поскольку структура слоя определяет функцию распределения ионов (ФРИ), бомбардирующих стенку, данная задача имеет большое значение для различных приложений, среди которых: вопросы исследования плазмохимических реакций, идущих с участием ионов; определение подвижности ионов в плазменном объекте; процессы нагрева нейтральной компоненты плазмы; современные плазменные нанотехнологии: тонкая очистка ионами поверхности изделий, технология создания рельефов на поверхности за счет избирательного травления при бомбардировке потоками ионов [1-4] и ряд других. Знание структуры пристеночного слоя необходимо и в теории зондов при определении концентрации заряженных частиц в плазме по так называемому току насыщения [5,6]. Отметим, что кинетическая теория для вычисления этого тока, использующая известный критерий Бома [7-10], также основывается на знании ФРИ в возмущенном слое и до сих пор окончательно не разработана [8]. Имея ввиду цель работы - вычисление на основе кинетической теории параметров ионного потока в возмущенном пристеночном слое (ВПС) при произвольных параметрах газового разряда, мы не будем здесь затрагивать вопросы численного моделирования процессов в ВПС (см., например, [11]), поскольку это, по-видимому, имеет смысл для конкретных условий в плазме, но не для поиска общих закономерностей.

Принято считать, что ВПС в рассматриваемой ситуации имеет следующую структуру (рис. 1). Между невозмущенной плазмой и пристеночным слоем (ПС), в котором существенно нарушение квазинейтральности, расположен так называемый квазинейтральный предслой, в котором и происходит ускорение ионов до скорости, обеспечивающей монотонность потенциала в ПС [8-10,12].Условие, которое определяет эту скорость, называется критерием Бома [7] (хотя сам Бом рассматривал только бесстолкновительный ВПС), а граничная точка между предслоем и ПС - точкой Бома. Отметим, что в общем случае соотношение протяженности предслоя, ПС и длины пробега ионов относительно перезарядки может быть произвольным. В предслое, поскольку он квазинейтрален, устанавливается слабо растущее электрическое поле, большее соответствующей величины $E$ в невозмущенной плазме, для того, чтобы ионы в нем ускорялись. При этом считается, что ближняя к стенке граница предслоя соответствует 


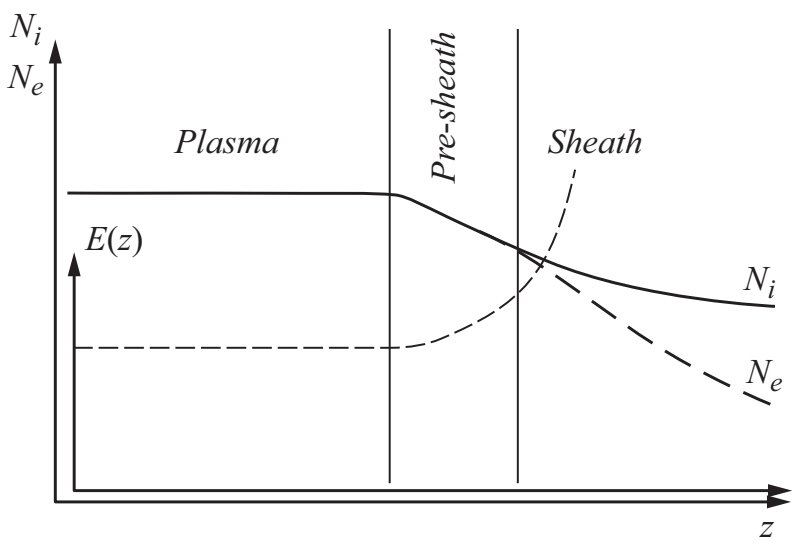

Рис. 1. Схематичное изображение структуры призондового слоя около стенки под большим по сравнению с температурой электронов отрицательным потенциалом.

потенциалу порядка средней энергии электронов [5]. Как будет видно в дальнейшем, кинетическое рассмотрение данной задачи с использованием реальной ФРИ и учетом процессов резонансной перезарядки в ПС приводит к выводу, что потенциал на внешней границе предслоя существенно зависит от параметров невозмущенной плазмы и может быть как больше, так и существенно меньше средней энергии электронов.

Под критерием Бома обычно понимают необходимость выполнения на границе, „предслоя“ и ПС в гидродинамическом приближении неравенства:

$$
\left|\frac{\partial n_{i}}{\partial U}\right| \leq\left|\frac{\partial n_{e}}{\partial U}\right|
$$

— в ,слабой“ форме $[7,13]$ и

$$
\frac{1}{n_{i}}\left|\frac{\partial n_{i}}{\partial U}\right| \leq \frac{1}{n_{e}}\left|\frac{\partial n_{e}}{\partial U}\right|
$$

- в „сильной“ $[14,15]$, где $n_{i}, n_{e}-$ концентрации ионов и электронов соответственно; $U$ - потенциал пространства в ВПС (при этом потенциал на границе невозмущенной плазмы и ВПС считается равным нулю). Известно также несколько формулировок кинетического критерия Бома, как, например, [16-19]:

$$
\frac{1}{2 n_{i}} \int_{0}^{\infty} w^{-3 / 2} f_{i}(U, w) d w \leq 1,
$$

и ряд других $[14,17]$ (где $w$ - отношение энергии иона к температуре электронов; $f_{i}\left(U, E_{i}\right)-$ ФРИ; $E_{i}-$ энергия иона). Кроме того, существуют формулировки кинетического критерия Бома, учитывающие возможный немаксвелловский характер ФРЭ. Выполнение неравенств (1)-(3) требуется для монотонности потенциала в ПС и приводит к тому, что средняя скорость ионов в точке Бома должна удовлетворять неравенству

$$
v_{i} \geq \psi\left(\frac{r_{d}}{\lambda_{i}}\right) \sqrt{\frac{k T_{e}}{M}},
$$

где $r_{d}, \lambda_{i}$ - радиус Дебая и длина пробега иона относительно процесса резонансной перезарядки; $T_{e}$ - температура электронов; $M$ - масса иона; $k$ - постоянная Больцмана; $\psi\left(r_{d} / \lambda_{i}\right)$ - некоторая функция, для которой справедливо $\psi\left(r_{d} / \lambda_{i}\right) \rightarrow 1$ при $r_{d} / \lambda_{i} \rightarrow 0$ [11,20-22].

Подробные обзоры работ с анализом различных теорий и принятых в работах допущений по данной тематике приведены в $[8,17,23]$. Мы не будем здесь проводить столь же тщательный анализ. Элементы модели, которую мы рассмотрим, уже применялись ранее другими авторами $[16,23]$.

Необходимость в критерии Бома, по-видимому, обусловлена отсутствием теории, описывающей параметры потока ионов в квазинейтральном предслое. Более или менее адекватные теории, учитывающие, в частности, перезарядку и ионизацию в ПС, построены для собственно ПС, однако для самосогласованного решения данной задачи в замкнутом виде требуются граничные условия, которые и ставятся, в частности, на границе ПС и предслоя. Напомним, что по причинам, изложенным выше, мы здесь не рассматриваем методы численного моделирования, в том числе и с применением алгоритмов Монте-Карло.

В отсутствие информации о ФРИ на этой границе для преодоления данной трудности ряд авторов предлагают разные способы сшивки решения для ПС и предслоя [24-27], а также делают различные предположения о поведении поля на границе ПС и предслоя, например, равенство нулю производной и (или) самого поля [22,23]. Ниже мы покажем, что подобные допущения приводят к существенному искажению структуры ВПС (особенно предслоя), которая зависит от средней энергии ионов в невозмущенной плазме даже при условии, что она много меньше таковой для электронов.

Интересно отметить, что Riemann в своей обзорной работе [8] прямо пишет, что „... в настоящее время не существует кинетического решения задачи в приближении „горячих“ ионов“ (т. е. с учетом не нулевой средней энергии ионов в невозмущенной плазме).

Критерий Бома, в частности, применяют для вычисления так называемого ионного тока насыщения на зонд, при этом им пользуются и в случае, когда не выполняется условие $r_{d} / \lambda_{i} \rightarrow 0$ [5], хотя первоначально Бом вывел свой критерий (соотношение $(4)$ при $\psi\left(r_{d} / \lambda_{i}\right)=1$ ) для бесстолкновительного ПС [7,28]:

$$
\kappa_{1}=\frac{r_{d}}{\lambda_{i}} \ll 1
$$

Еще один источник погрешностей при описании предслоя - не учет реальной ФРИ в невозмущенной плазме, особенно, в ситуации, когда энергия ионов 
достаточно велика (см., например, [29]). Как уже упоминалось, до сих пор авторы, исследуя известный критерий Бома, полагали ФРИ на границе возмущенного слоя около поверхности при отрицательном относительно плазмы потенциале либо в виде дельта-функции при некоторой средней скорости ионов (обычно нулевой) $[15,20,21,30-32]$, либо, не конкретизируя выражение ФРИ [19] при нулевой энергии ионов в плазме, используя кинетический подход, пытались получить общее соотношение. Следует отметить, что в кинетическом подходе без конкретизации вида ФРИ, по-видимому, теряется важная информация, поскольку, как легко видеть, количественное выражение критерия Бома существенно зависит от вида ФРИ.

Отметим, что, как показано в работах [29,33-37], ФРИ в слабоионизованной плазме постоянного тока имеет слабо выраженный максимум в области низких энергий (порядка тепловой скорости атомов) и далее достаточно медленно (особенно при сильных полях) спадает в область больших энергий. Отсюда следует, что допущения о „средней скорости“, используемые в гидродинамическом приближении $[12,20,21,30,31]$ и при аппроксимации ФРИ дельта-функцией, неудовлетворительно описывают ситуацию. Наконец, применение модели с нулевой средней энергией ионов в плазме нарушает непрерывность электрического поля на границе предслоя и невозмущенной плазмы, поскольку требует либо равенства нулю длины пробега ионов, либо равенства нулю электрического поля в невозмущенной плазме. Известные модели Lewi Tonks и Irving Langmuir [38], в которых эти предположения отсутствуют, предполагают существование бесстолкновительного ВПС.

\section{Построение теории ВПС}

Будем основываться на развитой в работе [39] теории расчета ФРИ в ВПС. Предположим, что в газоразрядной плазме есть плоская поверхность, находящаяся под некоторым отрицательным относительно плазмы потенциалом $U$. Тогда вблизи этой поверхности образуется ВПС. Пусть на некотором расстоянии от стенки параметры плазмы мало отличаются от таковых для случая $U=0$. Будем считать эту плоскость границей невозмущенной плазмы и ВПС. Введем систему прямоугольных декартовых координат, таким образом, что плоскость $X Y$ этой системы совпадает с введенной выше плоской границей. Качественно структура ВПС, согласно вышесказанному, имеет вид, представленный на рис. 1.

Сначала допустим, что функция распределения электронов (ФРЭ) - максвелловская, а сечение перезарядки не зависит от энергии иона. Тогда, согласно результатам работы [39], структура ВПС при постоянном сечении резонансной перезарядки иона определяется из решения системы уравнений:

$$
R^{\prime}(V)=\frac{\kappa_{2}}{\sqrt{F(V)}},
$$

$F^{\prime}(V)=\frac{2 \kappa_{2}^{2}}{\kappa_{1}^{2}}$

$$
\begin{gathered}
\times\left\{\exp \left[-R(V)+\kappa_{2} V\right] \operatorname{erfc}\left(\sqrt{\kappa_{2} V}\right)+Q(V)-\exp (-V)\right\}, \\
R(0)=0, \quad F(0)=1, \quad F(V)=\left[\frac{1}{a(V)}\right]^{2},
\end{gathered}
$$$$
Q(V)=\sqrt{\frac{\kappa_{2}}{\pi}} \exp [-R(V)] \int_{0}^{V} \exp \left[R\left(v^{\prime}\right)\right] \frac{F^{-0.5}\left(v^{\prime}\right) d v^{\prime}}{\sqrt{V-v^{\prime}}},
$$

где $\kappa_{2}=\frac{k T_{e}}{e E \lambda_{i}}, V=\frac{|e U|}{k T_{e}} \geq 0, a(V)=E / E(V), E(V)-$ электрическое поле в ВПС и $E(0)=E-$ электрическое поле в невозмущенной плазме. Зависимость относительной концентрации ионов $\bar{N}_{i}=\frac{n_{i}}{n_{0}}$ (где $n_{0}-$ концентрация заряженных частиц в невозмущенной плазме) от потенциала в ВПС дается соотношениями:

$$
\bar{N}_{i}(V)=\frac{\kappa_{1}^{2}}{2 \kappa_{2}^{2}} F^{\prime}(V)+\exp (-V), \quad \bar{N}_{i}(0)=1 .
$$

В случае, когда сечение перезарядки зависит от энергии иона:

$$
\sigma\left(E_{i}\right)=\sigma_{0} B\left(E_{i}\right),
$$

где $B\left(E_{i}\right)$ - некоторая известная функция, решать систему (6) можно методом последовательных приближений (см. Приложение). Зависимость сечения резонансной перезарядки от энергии относительного движения иона и атома (а в нашем случае быстрых по сравнению с атомами ионов - от энергии иона) хорошо аппроксимируется выражением

$$
B\left(E_{i}\right)=\left[1-a \cdot \ln \left(E_{i}\right)\right]^{2},
$$

где, например, для $\mathrm{Ar}$ численный коэффициент $a=0.0543$ [40], если $E_{i}$ выражена в $\mathrm{eV}$.

Переменные $R(V), F(V)$ имеют простой физический смысл. Первая - это зависимость координаты в ВПС (в единицах длины пробега иона относительно перезарядки), отсчитываемая от границы невозмущенной плазмы как функция безразмерного потенциала $V$. Вторая - это квадрат относительного электрического поля. В соответствии с этим формулируются и граничные условия к системе (6). Таким образом, зависимость потенциала $V$ от безразмерной координаты $Y$ есть обратная функция $R(V)$, а зависимость электрического поля в ВПС от безразмерной координаты выражается через $F(V)$ :

$$
V(Y)=R^{-1}(V), \quad E(Y)=E \cdot F^{-0.5}[V(Y)] .
$$

Интересно также исследовать зависимость структуры ВПС от вида функции распределения электронов при их 
одинаковой средней энергии. Для примера возьмем две $\Phi \mathrm{PЭ}-f_{e d}\left(E_{i}\right), f_{e h c}\left(E_{i}\right):$

$$
\begin{gathered}
f_{e d}\left(E_{e}\right)=\frac{1}{2 \Gamma(3 / 4)\left(k T_{e d}\right)^{1.5}} \cdot \exp \left[-\left(\frac{E_{e}}{k T_{e d}}\right)^{2}\right] \\
f_{e h c}\left(E_{e}=\frac{1}{4\left(k T_{e h c}^{1.5}\right.} \cdot \exp \left(-\sqrt{\frac{E_{e}}{k T_{e h c}}}\right) .\right.
\end{gathered}
$$

Для равенства средних энергий электронов в случае максвелловской ФРЭ и определенных соотношениями (11) необходимо выполнение равенств

$$
T_{e d} \approx 2.03 \cdot T_{e} ; \quad T_{e h c}=0.125 \cdot T_{e}
$$

Первая из введенных функций - дрювистейновская обладает меньшим количеством быстрых электронов по сравнению с максвелловской, вторая же, напротив, имеет быстрых электронов больше максвелловской, как, например, ФРЭ в тлеющем разряде с полым катодом $[41,42]$.

Концентрация электронов для ФРЭ $f_{e d}\left(E_{i}\right)$ и $f_{e h c}\left(E_{i}\right)$ выражается соотношениями

$$
\begin{gathered}
N_{e d}(V)=\frac{2}{\Gamma\left(\frac{3}{4}\right)} \int_{0}^{\infty} \sqrt{\varepsilon^{\prime}} \exp \left[-\left(\varepsilon^{\prime}+\frac{V}{2.03}\right)^{2}\right] d \varepsilon, \\
N_{e h c}(V)=0.25 \int_{0}^{\infty} \sqrt{\varepsilon^{\prime}} \exp \left[-\sqrt{\varepsilon^{\prime}+8 V}\right] d \varepsilon
\end{gathered}
$$

соответственно. Таким образом, для исследования структуры ВПС при немаксвелловской ФРЭ необходимо в правой части второго уравнения системы (6) и в соотношении (7) заменить $\exp (-V)$ на $N_{e d}(V)$ или $N_{e h c}(V)$, определенные формулами (14). Параметр же $\kappa_{2}$ остается неизменным и определяется через параметры $T_{e d} / 2.03$ и $8 T_{e h c}$ (оба равны $T_{e}$ ).

Используя полученную в работе [39] ФРИ, которая нормирована на поток ионов, нетрудно получить выражение для средней скорости ионов при безразмерном потенциале $V$ :

$$
v_{i}(V)=v_{B} \frac{1}{\bar{N}_{i}(V)} \sqrt{\frac{2}{\pi \kappa_{2}}},
$$

где $v_{B}=\sqrt{\frac{k T_{e}}{M}}-$ бомовская скорость [7]. При этом для плотности потока ионов на плоскую стенку бесконечных размеров в отсутствие ионизации в ВПС выполняется:

$$
v_{i}(V) n_{i}(V)=n_{0} \sqrt{\frac{2 e E \lambda_{i}}{\pi M}} .
$$

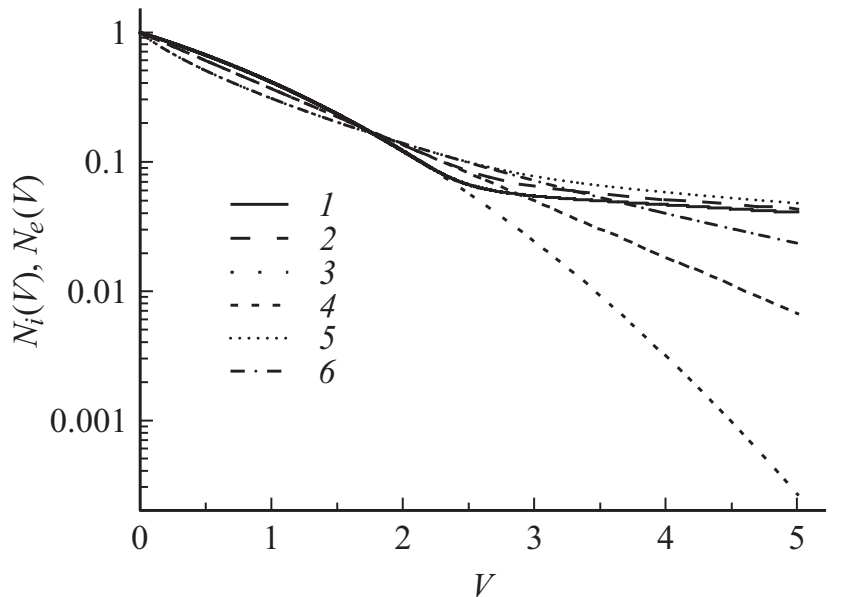

Рис. 2. Зависимость концентраций заряженных частиц в ВПС от относительного потенциала, рассчитанные для ФРЭ Максвелла и $f_{e d}\left(E_{i}\right), f_{e h c}\left(E_{i}\right)$ (формулы $\left.(11)\right) ; 1,3-N_{i}(V)$, $N_{e d}(V)$ для ФРЭ $f_{e d}\left(E_{i}\right)$ соответственно; $2,4-N_{i}(V), N_{e}(V)$ для ФРЭ Максвелла соответственно; 5, $6-N_{i}(V), N_{\text {ehc }}$ для ФРЭ $f_{\text {ehc }}\left(E_{i}\right) ; \kappa_{1}=0.003 ; \kappa_{2}=70$.

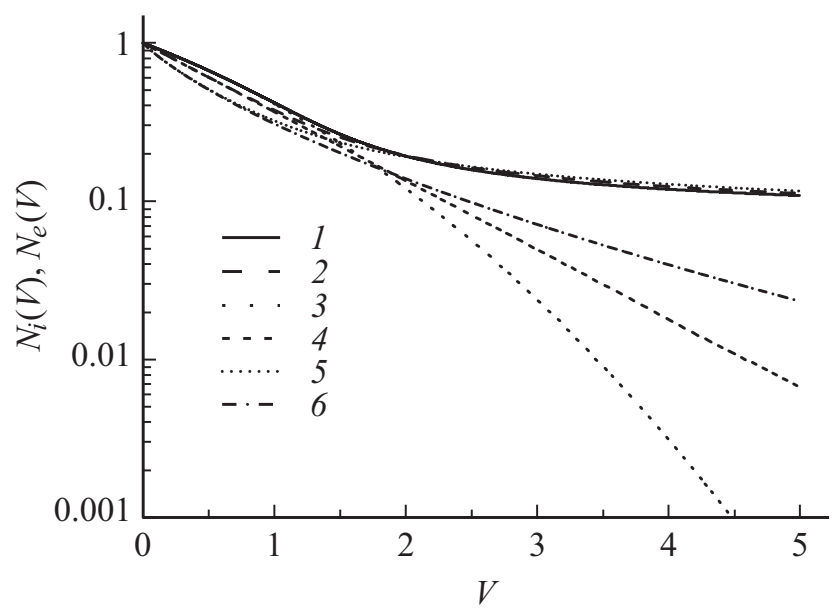

Рис. 3. То же, что и на рис. 2 , но для $\kappa_{1}=1 ; \kappa_{2}=100$.

\section{Обсуждение полученных результатов}

На рис. 2,3 приведены результаты расчетов концентрации ионов и электронов для параметров $\kappa_{1}=0.003$, $\kappa_{2}=70$ и $\kappa_{1}=1, \kappa_{2}=100$ соответственно для максвелловской и определенных формулами (11), (12) ФРЭ для случая, когда зависимость сечения перезарядки от энергии иона определяется формулой (9) с параметром $a=0.0543$ (для Ar). Во первых, как мы видим из представленных данных, при любой ФРЭ ВПС действительно разделяется на два существенно различных по своим свойствам слоя - квазинейтральный предслой и ПС, где квазинейтральность существенно нарушается. При малом значении параметра $\kappa_{1}$ наблюдается некоторое расхождение в поведении концентраций ионов в области границы предслоя и ПС в зависимости от вида ФРЭ, в то 


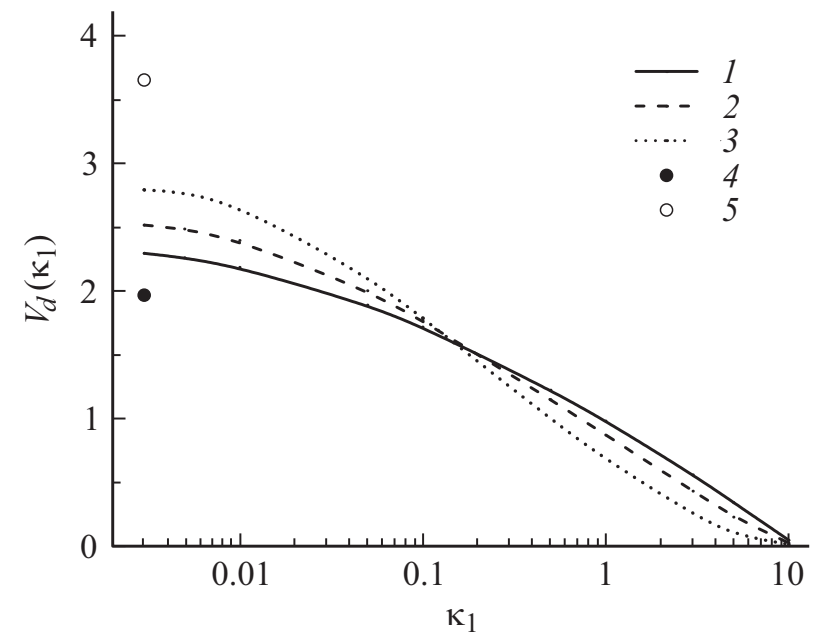

Рис. 4. Зависимость относительного потенциала на границе предслоя и ПС от параметра $\kappa_{1}$ для: 1 - ФРЭ $f_{e d}\left(E_{i}\right)$, $\kappa_{2}=100 ; 2-$ ФРЭ Максвелла, $\kappa_{2}=100 ; 3-$ ФРЭ $f_{\text {ehc }}\left(E_{i}\right)$, $\kappa_{2}=100 ; 4-$ ФРЭ Максвелла, $\kappa_{1}=0.003, \kappa_{2}=20 ; 5-$ ФРЭ Максвелла, $\kappa_{1}=0.003, \kappa_{2}=1000$.

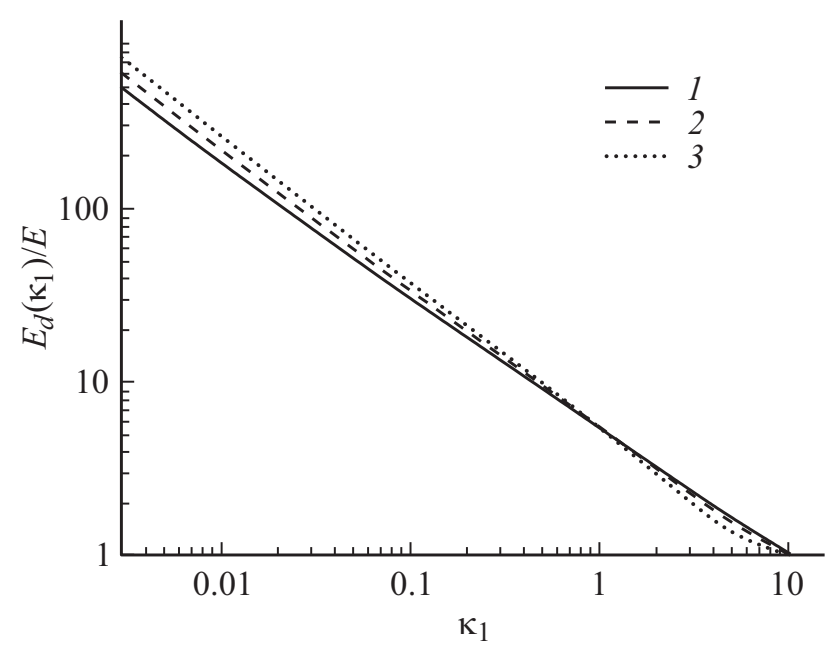

Pис. 5. Зависимость относительного электрического поля на границе предслоя и ПС от параметра $\kappa_{1}$ для: $1-\Phi Р Э ~ f e d\left(E_{i}\right)$; 2 - ФРЭ Максвелла; $3-$ ФРЭ $f_{\text {ehc }}\left(E_{i}\right) ; \kappa_{2}=100$.

время как для $\kappa_{1}=1$ отличия в поведении этих величин для ионов существенно меньше. Это, видимо, связано с тем, что малое значение параметра $\kappa_{1}$, соответствующее небольшому радиусу Дебая, приводит к большему значению электрического поля при одинаковой разнице в концентрациях ионов и электронов.

На рис. 4 приведена зависимость относительного потенциала $V_{d}=\frac{\left|e U_{d}\right|}{k T_{e}}$ на границе квазинейтрального „предслоя“ и ПС от параметра $\kappa_{1}$ при $\kappa_{2}=100$ для случаев ФРЭ Максвелла и определенных формулой (11), а также $V_{d}$ при $\kappa_{1}=0.003$ для $\kappa_{2}=20$ и 1000. При этом также учитывалась зависимость сечения перезарядки от энергии иона (формула (9) с параметром $a$ для $\mathrm{Ar}$ ), a потенциал $V_{d}$ определялся из численного решения уравнения

$$
\left|\frac{\partial n_{i}}{\partial V_{d}}\right|=\left|\frac{\partial n_{e}}{\partial V_{d}}\right|
$$

при контроле за соблюдением неравенства $\left|\frac{\partial n_{i}}{\partial U}\right|<\left|\frac{\partial n_{e}}{\partial U}\right|$, если $V<V_{d}<0$. Видно, что результаты расчетов для всех ФРЭ близки. Кроме того, при $\kappa_{1} \ll 1$ потенциал $V_{d}$ зависит от параметра $\kappa_{2}$. Таким образом, предположение о том, что при $\kappa_{1} \ll 1$ на границе „предслоя“ и ПС потенциал близок к величине $k T_{e}$, может приводить к существенным погрешностям при вычислении ионного тока насыщения с использованием критерия Бома. Отметим, что, как показывают расчеты, протяженность предслоя, выраженная в длинах свободного пробега ионов, при всех параметрах $\kappa_{1}$ и $\kappa_{2}=100$ существенно превосходит единицу.

Из данных рис. 5 видно, что величина относительного электрического поля на границе предслоя и ПС, рассчитанного для ФРЭ Максвелла, несколько выше аналогичной величины, вычисленной для ФРЭ $f_{e d}\left(E_{e}\right)$, и ниже определенного для ФРЭ $f_{e h c}\left(E_{e}\right)$. Это можно объяснить тем, что в предслое, где величина потенциала не слишком велика, концентрация электронов $N_{e d}(V)$ с ростом потенциала спадает медленнее, чем $\exp (-V)$ и тем более, чем $N_{e h c}(V)$. Соответственно этому нарушение квазинейтральности в случае $f_{e d}(\varepsilon)$ менее выражено, что ведет к более низкому значению электрического поля в предслое. Напротив, в ПС, где потенциал $V$ достигает существенной величины, концентрация, рассчитанная для ФРЭ Максвелла, спадает медленнее, чем $N_{e d}(V)$ и быстрее, чем $N_{e h c}(V)$. В силу этого вычисленные значения электрического поля для двух рассматриваемых случаев имеют обратное соотношение. Это иллюстрируют данные рис. 6, где представлены зависимости относительного электрического

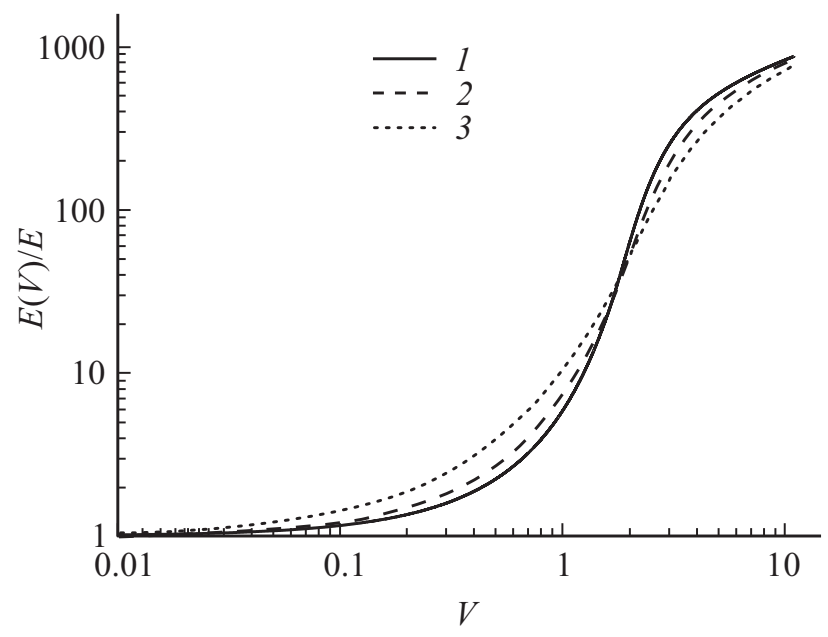

Рис. 6. Зависимости относительного электрического поля в ВПС от потенциала, рассчитанные для: $1-\Phi Р Э ~ f_{e d}\left(E_{i}\right)$; 2 - ФРЭ Максвелла; $3-$ ФРЭ $f_{\text {ehc }}\left(E_{i}\right) ; \kappa_{1}=0.1 ; \kappa_{2}=100$. 


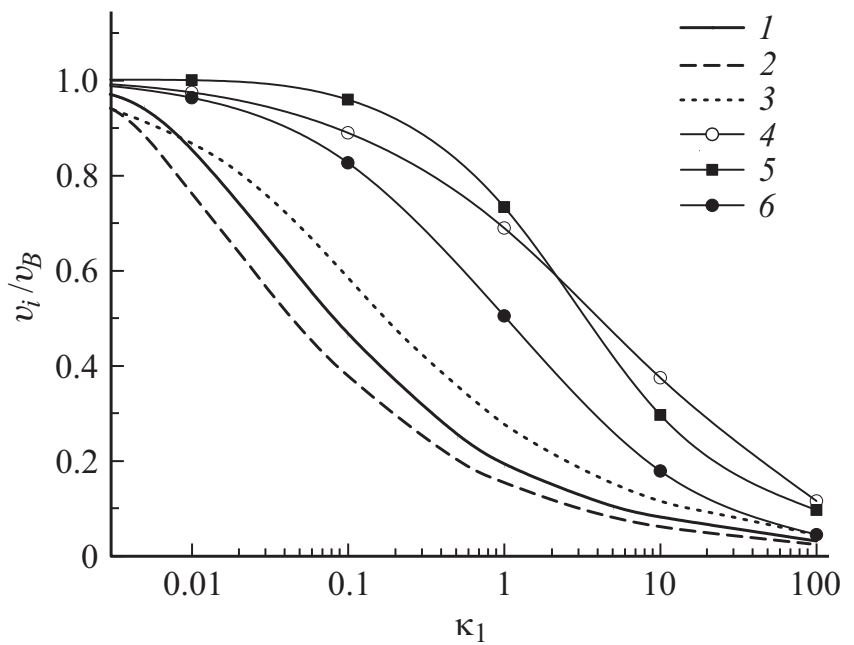

Рис. 7. Зависимость отношения средней скорости иона к бомовской скорости от параметра $\kappa_{1}$ на границе квазинейтрального предслоя и ПС; 1,2 - расчет по разработанной кинетической теории при $\kappa_{2}=100,1000$ соответственно; 3-6 - данные работ $[21,12,20,19]$ соответственно, вычисленные в гидродинамическом приближении; в работе [21] скорость ионов в плазме считалась нулевой; в работах $[12,20,19]$ считалось, что все ионы имеют на границе предслоя некоторую одинаковую скорость и структура предслоя не рассматривалась.

поля от относительного потенциала в ВПС для $\kappa_{1}=0.1$, $\kappa_{2}=100$, рассчитанные для трех рассматриваемых ФРЭ.

На рис. 7 приведено сравнение зависимости средней скорости ионов в точке Бома $\frac{v_{i}}{v_{B}}\left(\kappa_{1}\right)$, рассчитанной авторами [11,20-22] в рамках различных модификаций гидродинамического подхода и вычисленной по разработанной нами теории для значений $\kappa_{2}=100,1000$. Видно, что наши расчеты для точки, где равны производные концентраций, наиболее близки к данным [22].

Данные, представленные на рис. 8, свидетельствуют о незначительном изменении средней скорости ионов в зависимости от вида ФРЭ при сохранении их средней энергии.

В целом можно констатировать, что структура ВПС слабо зависит от вида ФРЭ при условии равенства их средних энергий.

Рассмотрим теперь результаты сравнения расчетов по нашей теории с экспериментальными данными других авторов. Сначала напомним, что в наших обозначениях ширина квазинейтрального предслоя равна $Y_{d}=Y\left(V_{d}\right)=$ $=R\left(V_{d}\right)$, а ширина ПС $-Y_{w}-Y_{d}=R\left(V_{w}\right)-R\left(V_{d}\right)$.

Обратимся к экспериментальным данным работы [43], в которой были измерены ФРИ, бомбардирующих поверхность плоской стенки в зависимости от отрицательного относительно плазмы потенциала при различных плазменных условиях в $\mathrm{Ar}, \mathrm{N}_{2}$ и $\mathrm{O}_{2}$. Кроме того, авторы на основе полученных экспериментальных данных определили ряд характеристик ионного потока вблизи стенки. Мы рассмотрим только данные для Ar, поскольку в молекулярных газах возможно образование различных ионов, что влияет на структуру ВПС, и не было учтено в нашей теории.

На рис. 9 приведено сравнение зависимости величины $V_{w} \cdot\left(\frac{4 \kappa_{1}^{2}}{9 j_{+}}\right)^{2 / 3}$ при $j_{+}=0.88161$ от относительной протяженности ПС $Y_{w}-Y_{d}$ при следующих условиях в плазме: $\mathrm{Ar} ; n_{0}=3.8 \cdot 10^{9} \mathrm{~cm}^{-3} ; k T_{e}=0.39 \mathrm{eV} ; P=3 \mathrm{~Pa}$. Соответствующая этим значениям величина параметра $\kappa_{1}=0.022$. Ширина ПС в эксперименте менялась посредством изменения потенциала стенки. Надо отметить, что, строго говоря, эти условия в невозмущенной плазме не соответствуют условиям применимости нашей теории, поскольку не выполняется условие сильного поля в невозмущенной плазме и средняя энергия ионов в ней оказывается порядка тепловой энергии атомов.

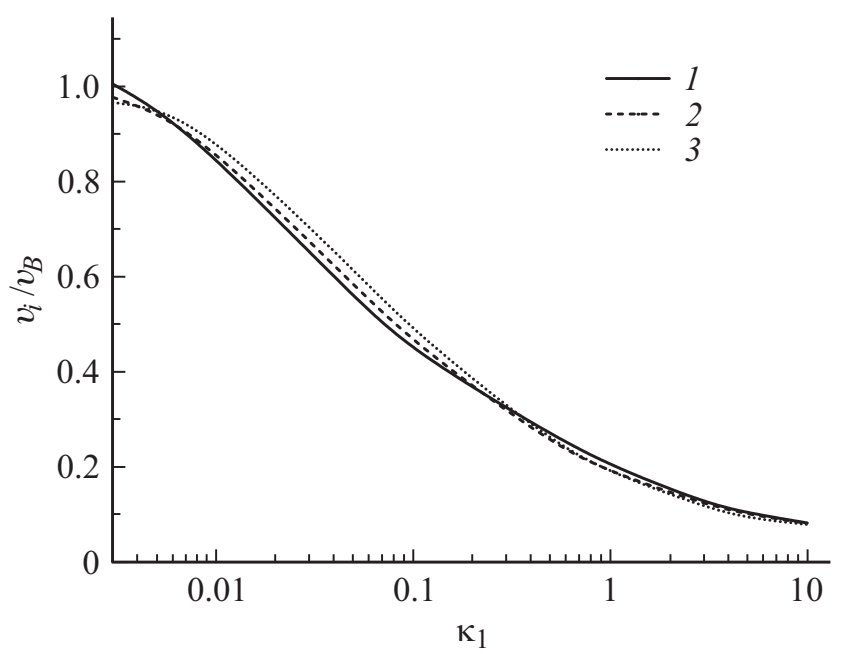

Рис. 8. Зависимость относительной средней скорости ионов на границе предслоя и ПС от параметра $\kappa_{1}$ для: $1-$ ФРЭ $f_{e d}\left(E_{i}\right) ; 2-$ ФРЭ Максвелла; $3-$ ФРЭ $f_{e h c}\left(E_{i}\right) ; \kappa_{2}=100$.

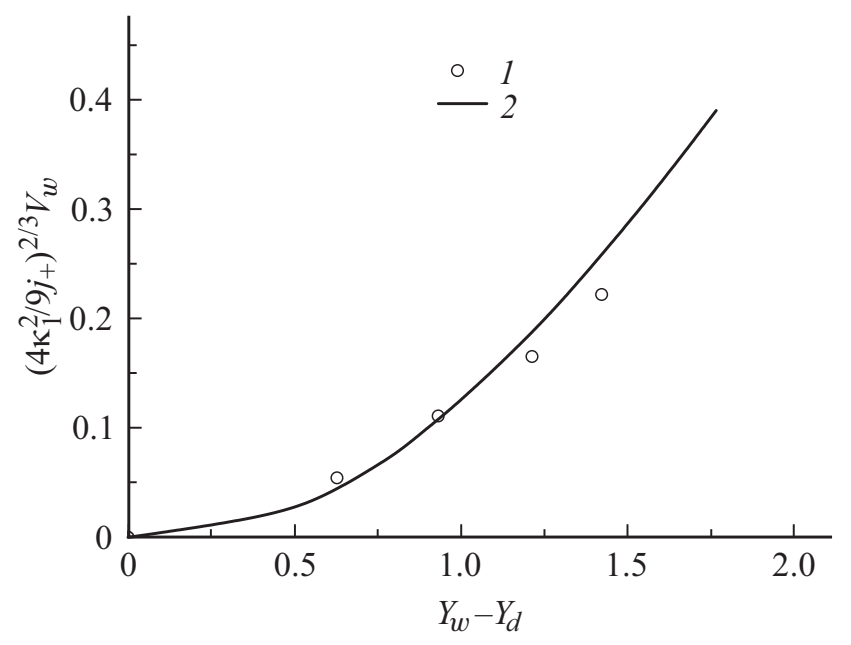

Рис. 9. Зависимость величины $V_{w} \cdot\left(4 \kappa_{1}^{2} / 9 j_{+}\right)^{2 / 3}$ от ширины ПС $Y_{w}-Y_{d}$ для условий экспериментов работы [43]: $j_{+}=0.88161 ; \operatorname{Ar} ; n_{0}=3.8 \cdot 10^{9} \mathrm{~cm}^{-3} ; k T_{e}=0.39 \mathrm{eV} ; P=3 \mathrm{~Pa} ;$ $\kappa_{1}=0.022 ; 1-$ экспериментальные данные данной работы; $2-$ наш расчет для $\kappa_{1}=0.022$. 


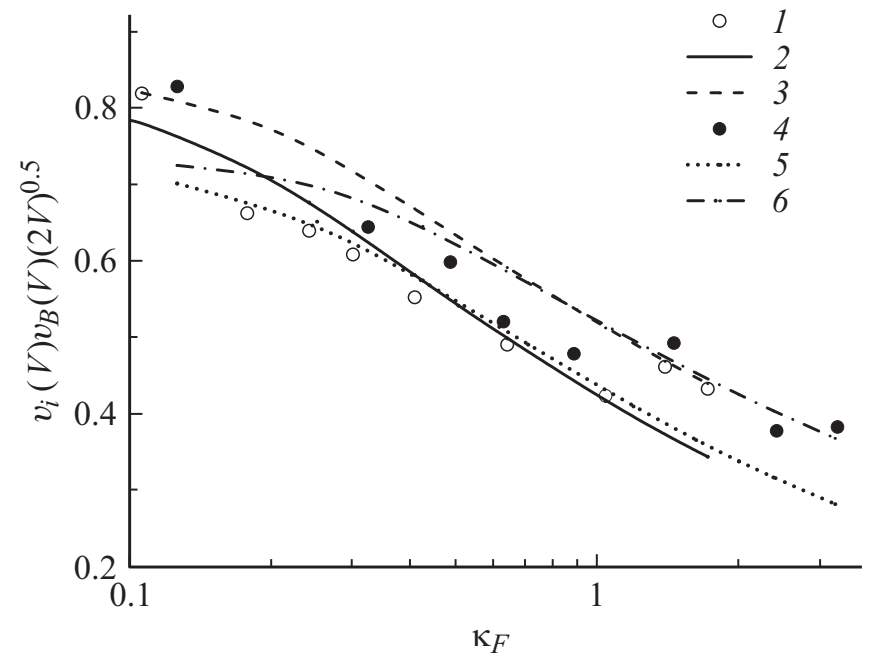

Рис. 10. Зависимость отношения $v_{i} / \sqrt{2 V}$ от параметра $k_{F}=V_{w}^{3 / 4}\left(4 \kappa_{1}^{2} / 9 j_{+}\right)^{1 / 2} ; 1-$ экспериментальные данные работы [43] для условий рис. 9; 2, 3 - наш расчет для этих же условий при $\kappa_{1}=0.022$ без учета зависимости сечения перезарядки от энергии иона и с учетом соответственно; $4-$ экспериментальные данные работы [43] для условий: $\mathrm{Ar} ; n_{0}=2 \cdot 10^{9} \mathrm{~cm}^{-3}$; $k T_{e}=0.46 \mathrm{eV} ; P=5 \mathrm{~Pa} ; \kappa_{1}=0.059 ; 5,6$ - наш расчет для этих же условий при $\kappa_{1}=0.059$ без учета зависимости сечения перезарядки от энергии иона и с учетом соответственно.

Поскольку авторы [43] не приводят данных о величине электрического поля в плазме, то мы взяли параметр $\kappa_{2}=25$, что соответствует энергии, которую ион набирает на длине пробега до ион- атомного столкновения с перезарядкой, примерно в два раза меньше тепловой энергии атомов при комнатной температуре. Отметим, что результаты расчетов слабо зависят от этого параметра. Из данных рис. 9 видно, что наблюдается удовлетворительное согласие наших расчетов с экспериментальными данными [43]. Некоторое расхождение при больших $Y_{w}-Y_{d}$ может быть связано, в том числе с экспериментальной ошибкой определения ширины ПС. При расчетах мы в этой части работы не учитывали зависимость сечения резонансной перезарядки от энергии иона, поскольку авторы при расчете величины $Y_{w}-Y_{d}$ из экспериментально измеренной ширины ПС также считали длину пробега постоянной.

Отметим, что для аналогичных по точности описания этих экспериментальных данных расчетов по собственной теории авторам пришлось в 5 раз увеличить параметр $\kappa_{1}$ (в цитируемой работе он обозначен как $\epsilon$ ) и проводить расчеты при $\kappa_{1}=0.11$. Мы же использовали $\kappa_{1}$, рассчитанный из экспериментальных условий настоящей работы.

На рис. 10 приведена зависимость экспериментально измеренной в [43] средней относительной скорости ионов, бомбардирующих стенку $\frac{v_{i}\left(V_{w}\right)}{v_{B} \sqrt{2 V_{w}}}$, от параметра $k_{F}=V_{w}^{3 / 4}\left(\frac{4 \kappa_{1}^{2}}{9 j_{+}}\right)^{1 / 2}$ для различных условий в плаз- ме Ar: условий рис. 9 и $n_{0}=2 \cdot 10^{9} \mathrm{~cm}^{-3} ; k T_{e}=0.46 \mathrm{eV}$; $P=5 \mathrm{~Pa} ; \kappa_{1}=0.059$ (величина параметра $\kappa_{1}$ для давления $P=5 \mathrm{~Pa}$ вычислена на основании приведенных авторами [43] данных). Здесь же даны результаты наших расчетов для этих условий без учета зависимости от энергии сечения перезарядки от энергии иона $\mathrm{Ar}^{+}$и с учетом. Расчеты проводились без подгоночных параметров. Как видно из этих данных, теоретические и экспериментальные результаты хорошо согласуются.

Зависимость безразмерного потенциала от переменной $\frac{z^{\prime}}{r_{d}}$, где $z^{\prime}-$ расстояние до стенки, находящейся под потенциалом $V_{w}=10$, представлена на рис. 11. Даны результаты наших расчетов в приближении $\kappa_{2} \rightarrow \infty$ (приближение холодных ионов) и $\kappa_{1} \gg 1$ (столкновительный режим). Здесь же приведены структуры ПС, вычисленные в рамках гидродинамической модели [44], различных модификаций кинетического подхода при нулевой средней энергии ионов в плазме [8,23]. Видно, что наши результаты при $\kappa_{2}=20000, \kappa_{1}=1000$ достаточно близки к данным кинетического подхода в столкновительном режиме и модели с холодными ионами, полученным в работе [23]. Однако необходимо отметить, что увеличение $\kappa_{1}$ в два раза приводит к заметным изменениям структуры ПС, при этом наши данные для $\kappa_{2}=20000, \kappa_{1}=2000$ достаточно близки к результатам расчета по гидродинамической модели [44].

Таким образом, можно сделать вывод, что, во-первых, структура ВПС слабо зависит от вида ФРЭ при сохранении средней энергии электронов, во-вторых, что известные экспериментальные данные других авторов хорошо описываются нашей теорией.

Рассмотрим теперь, как структура ВПС зависит от величины средней энергии ионов в невозмущенной плазме по отношению к средней энергии электронов. Расчеты показывают, что при увеличении параметра $\kappa_{2}$

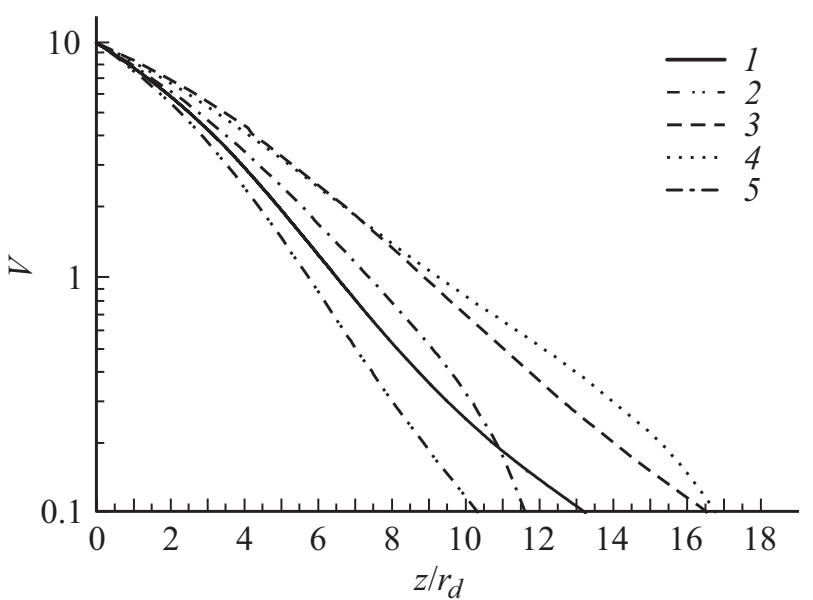

Рис. 11. Структура ПС, согласно различным моделям; 1 - гидродинамическая модель [43]; 2 - кинетическая модель [8]; 3 - СХ-модель [22]; 4, 5 - расчет по разработанной теории при $\kappa_{2}=20000 ; \kappa_{2} / \kappa_{1}=20,10$ соответственно; $z^{\prime}=0$ соответствует положению стенки при безразмерном потенциале $V=10$. 


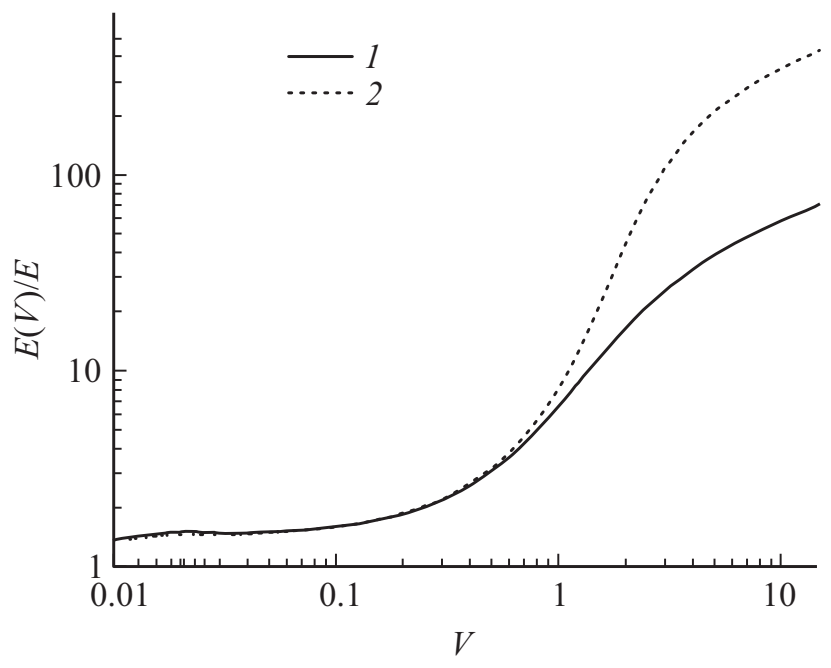

Рис. 12. Сравнение зависимости электрического поля в пристеночном слое от безразмерного потенциала, рассчитанной для тлеющего разряда в $\mathrm{He}$ в условиях работы [36]: $P=0.2$ Torr, $E=4 \mathrm{~V} / \mathrm{cm}, r_{D}=0.062 \mathrm{~cm}, \kappa_{1}=0.534$, $\kappa_{2}=20-1$ и для тех же условий, но при $\kappa_{2}=200-2$; при $\kappa_{2}=20-V_{d}=0.43$; при $\kappa_{2}=200-V_{d}=1.38$.

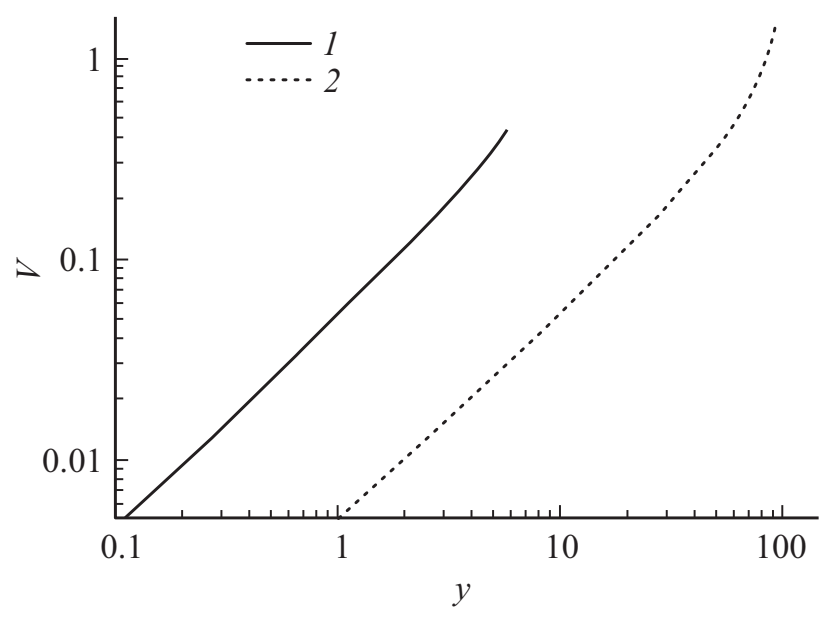

Pис. 13. Структура квазинейтрального предслоя для условий рис. $12 ; 1-\kappa_{2}=20 ; 2-\kappa_{2}=200$.

протяженность квазинейтрального предслоя $Y_{d}$ возрастает, при этом протяженность ПС $Y_{w}-Y_{d}$ практически не изменяется. При увеличении же параметра $\kappa_{1}$, напротив, протяженность квазинейтрального предслоя остается постоянной, а протяженность ПС растет. Рассмотрим физические причины такого поведения величин $Y_{d}$ и $Y_{w}-Y_{d}$. Основная роль предслоя - ускорение ионов до скорости, при которой обеспечивается монотонный характер потенциала в ПС. Увеличение параметра $\kappa_{2}$ означает либо уменьшение средней энергии ионов в невозмущенной плазме, либо рост средней энергии электронов. В обоих случаях необходимое для ускорения ионов расстояние возрастает. Слабая же зависимость $Y_{d}$ от параметра $\kappa_{1}$ обусловлена тем, что в силу квазинейтральности предслоя размеры радиуса Дебая по сравнению с длиной пробега ионов незначительно влияют на процесс ускорения ионов в предслое, а значит, и на его протяженность. В целом можно констатировать, что при любых параметрах $\kappa_{1}$ и $\kappa_{2} \gg 1$ протяженность квазинейтрального предслоя много больше длины пробега иона относительно перезарядки.

Что касается протяженности ПС $Y_{w}-Y_{d}$, то в этой части ВПС, напротив, величина средней энергии ионов в невозмущенной плазме по сравнению со средней энергией электронов практически не имеет значения, поскольку ионы в предслое ускоряются до некоторой скорости, существенно превосходящей начальную. Существенная зависимость $Y_{w}-Y_{d}$ от параметра $\kappa_{1}$ обусловлена тем, что структура слоя, где существенно нарушается квазинейтральность, определяется размером радиуса Дебая. Далее все вычисления будем производить для максвелловской ФРЭ.

На рис. 12 приведена рассчитанная нами зависимость относительного электрического поля в ВПС от безразмерного потенциала в условиях работы [36], когда параметр $\kappa_{2}=20$ и для $\kappa_{2}=200$. При расчетах учитывалась зависимость сечения перезарядки от энергии иона в виде (9) с параметром $a=0.0557$ для Не [40]. Изменение параметра $\kappa_{2}$ при сохранении значения параметра $\kappa_{1}$ соответствует уменьшению значения электрического поля в невозмущенной плазме. Таким образом, если в условиях работы [36] при $\kappa_{2}=20$ выполняется $E=4 \mathrm{~V} / \mathrm{cm}, V_{d}=0.43$, то при $\kappa_{2}=200$ поле в плазме равно $E=0.4 \mathrm{~V} / \mathrm{cm}$, а $V_{d}=1.38$. Как видно из данных рис. 12 , с ростом $\kappa_{2}$ увеличивается относительное электрическое поле в ВПС. Это, по-видимому, связано с тем, что при уменьшении средней энергии ионов в невозмущенной плазме требуется большее ускорение ионов для обеспечения монотонности потенциал в ВПС.

Важность учета средней энергии ионов в невозмущенной плазме для расчета структуры пристеночного слоя иллюстрируют рис. 13 и 14, на которых для

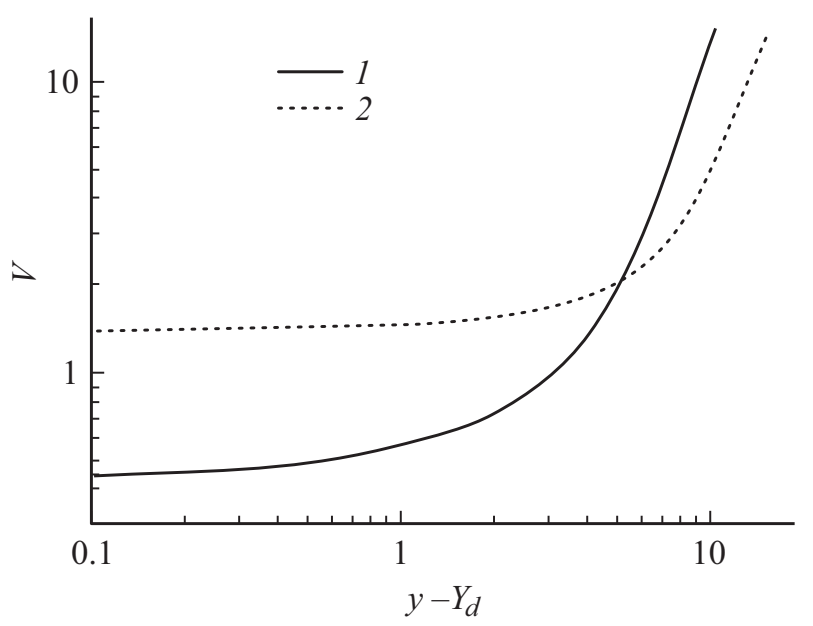

Рис. 14. Структура ПС для условий рис. $12 ; 1-\kappa_{2}=20$; $2-\kappa_{2}=200$. 
условий работы [36] в тлеющем разряде постоянного тока в Не представлены результаты расчетов структуры квазинейтрального предслоя $V(y)$ и ПС $V\left(y-Y_{d}\right)$, соответственно для $\kappa_{2}=20,200$. Видно, что с ростом $\kappa_{2}$ изменяется как структура „предслоя“, так и ПС.

Таким образом, можно сделать вывод о том, что учет средней энергии ионов в невозмущенной плазме важен даже при условии $\kappa_{2} \gg 1$.

\section{Заключение}

Основной недостаток существующих подходов построения общей теории ВПС - принятие упрощающих допущений (см. выше), затрудняющих корректное вычисление структуры квазинейтрального предслоя и параметров ионного потока в нем.

Именно поэтому для вычисления ионного тока насыщения на плоский зонд появляется необходимость в расчете скорости ионов на границе предслоя и ПС и соответственно в появлении критерия Бома. Однако корректное применение критерия Бома предусматривает знание функции $\psi\left(\frac{r_{d}}{\lambda_{i}}\right)$, т.е. концентрации электронов (входящей в формулу для радиуса Дебая), которая и подлежит определению. Как мы видели, в рассмотренной ситуации плотность ионного тока на бесконечную плоскую стенку остается неизменной при произвольных параметрах $\kappa_{1}, \kappa_{2}$. Для корректного использования результатов работы при зондовых измерениях плоским зондом необходимо учитывать изменение собирающей поверхности зонда при изменении его отрицательного потенциала [45-47].

Необходимо иметь ввиду, что, как следует из определения параметров $\kappa_{1}, \kappa_{2}$, при изменении длины пробега иона они изменяются оба. При изменении электрического поля в невозмущенной плазме изменяется только параметр $\kappa_{2}$.

При построении данной теории мы ввели границу невозмущенной плазмы и предслоя из условия равенства на ней ФРИ таковой в невозмущенной плазме. Кроме того, при решении уравнения Пуассона мы поставили условие для электрического поля на этой границе в виде его равенства полю в плазме. Ясно, что возмущение от стенки, находящейся под потенциалом, отличном от потенциала плазмы в этом месте, распространяется в плазму, строго говоря, на бесконечное расстояние. Однако в силу экранировки, оно убывает и на некотором расстоянии от стенки ФРИ становится близким к таковому в невозмущенной плазме, при этом производной электрического поля можно пренебречь.

В работе мы исследовали случай разряда постоянного тока при условии, что электрическое поле $\mathbf{E}$ в невозмущенной плазме направлено ортогонально стенке (или плоскому зонду), находящейся при отрицательном потенциале и для условий, когда можно пренебречь ионизацией в ВПС. Если же речь идет об ориентации электрического поля $\mathbf{E}$ под произвольным углом, или о разряде иного типа, например, RF-разряде, то конкретные формулы, конечно же, будут иными, но изложенный выше подход остается справедливым и может быть реализован. Этому и будет посвящена наша дальнейшая работа.

\section{Конфликт интересов}

Авторы заявляют, что у них нет конфликта интересов.

\section{Приложение}

Перейдем к относительной энергии иона $\varepsilon=\frac{E_{i}}{k T_{e}}$. Предположим, что зависимость сечения перезарядки от энергии иона определяется соотношением (7). Тогда система (6) записывается в виде [39]:

$$
R^{\prime}(V)=\frac{\kappa_{2} K(V)}{\sqrt{F(V)}}
$$

$$
\begin{aligned}
& F^{\prime}(V)=\frac{2 \kappa_{2}^{2}}{\kappa_{1}^{2}} \\
& \times\left\{\exp \left[-R(V)+\kappa_{2} V\right] \operatorname{erfc}\left(\sqrt{\kappa_{2} V}\right)+Q(V)-\exp (-V)\right\},
\end{aligned}
$$

$$
Q(V)=\sqrt{\frac{\kappa_{2}}{\pi}} \exp [-R(V)] \int_{0}^{V} \exp \left[R\left(v^{\prime}\right)\right] \frac{F^{-0.5}\left(v^{\prime}\right) B\left(v^{\prime}\right) d v^{\prime}}{\sqrt{V-v^{\prime}}} .
$$

Функция $K(V)$ является решением интегрального уравнения

$$
\begin{aligned}
K(V)= & \exp [-R(V)] \int_{0}^{\infty} B\left[2 \kappa_{2}(\varepsilon+V)\right] f_{i 0}(\varepsilon) d \varepsilon \\
& +\kappa_{2} \exp [-R(V)] \int_{0}^{V} F^{-0.5}\left(v^{\prime}\right) \exp \left[R\left(V^{\prime}\right)\right] \\
& \times B\left[2 \kappa_{2}\left(V-V^{\prime}\right)\right] K\left(V^{\prime}\right) d V^{\prime}
\end{aligned}
$$

и имеет простой физический смысл - это средняя по ФРИ при заданном потенциале $V$ величина функции $B(\varepsilon)$. Функция $f_{i 0}(\varepsilon)$ в правой части $(П 2)-$ это ФРИ в невозмущенной плазме. В приближении сильного поля, когда энергия иона, которую он приобретает на длине свободного пробега относительно перезарядки, много больше тепловой энергии атомов, согласно результатам [37], для нее справедливо

$$
f_{i 0}(\varepsilon)=C \exp \left(-\kappa_{2} \varepsilon\right) \operatorname{erfc}\left(-\sqrt{\left(\varepsilon_{0}-1\right) \kappa_{2} \varepsilon}\right),
$$

где

$$
C=\frac{I_{0}}{v_{i}^{2}\left(1+\sqrt{\frac{\varepsilon_{0}-1}{\varepsilon_{0}}}\right)} \approx \frac{I_{0}}{2 v_{i}^{2}}
$$


$I_{0}-$ плотность потока ионов, на которую нормирована ФРИ $f_{i 0}(v)$;

$$
\begin{gathered}
\operatorname{erfc}(-x)=\frac{2}{\sqrt{\pi}} \int_{x}^{\infty} \exp \left(-y^{2}\right) d y \\
\varepsilon_{0}=\frac{e E \lambda_{i}}{2 k T_{a}}, \quad v_{i}=\sqrt{\frac{e E \lambda_{i}}{M}} .
\end{gathered}
$$

Как показано в работе [39], при условии, что в ВПС (т.е. в квазинейтральном предслое и ПС) ион испытывает хотя бы одно столкновение (что реализуется практически при любых параметрах в плазме, поскольку как показывают расчеты, протяженность предслоя всегда более одной длины пробега иона), для уравнения (П2) справедливо решение:

$$
\begin{aligned}
& K(V) \equiv \bar{K}(V)+\left\{1-\kappa_{2} \exp [-R(V)] \int_{0}^{V} F^{-0.5}\left(V^{\prime}\right)\right. \\
& \left.\times \exp \left[R\left(V^{\prime}\right)\right] B\left[2 \kappa_{2}\left(V-V^{\prime}\right)\right] \bar{K}\left(V^{\prime}\right) d V^{\prime}\right\}\left\{1-\kappa_{2} \exp [-R(V)]\right. \\
& \left.\times \int_{0}^{V} F^{-0.5}\left(V^{\prime}\right) \exp \left[R\left(V^{\prime}\right)\right] B\left[2 \kappa_{2}\left(V-V^{\prime}\right)\right] d V^{\prime}\right\}^{-1},
\end{aligned}
$$

В качестве нулевого приближения решения системы (6) можно взять решение $R^{(0)}(V), F^{(0)}(V)$, когда для $K(V)$ используется $K^{(0)}(V)=B(V)$.

Для вычисления первого и последующих приближений сначала вычисляем $K^{(1)}(V)$, используя в правой части (П4) полученные ранее $R^{(0)}(V), F^{(0)}(V)$. Затем, решая (П2) с использованием найденной функции $K^{(1)}(V)$, находим $R^{(1)}(V), F^{(1)}(V)$ и т. д. Процедура практически для любых параметров $\kappa_{1}, \kappa_{2}$ сходится настолько быстро, что достаточно первого приближения. Для уменьшения времени расчетов можно пользоваться найденными в [39] приближенными формулами:

$$
\begin{aligned}
& Q(V) \equiv Q_{a}(V)=F^{-0.25 b(V)} \operatorname{erf}[\sqrt{R(V)}] ; \\
& b(V)=1 \quad \text { если } \quad V<a_{1} ; \\
& b(V)=1-5\left[\left(\frac{V}{a_{1}}\right)^{0.075}\left(\frac{a_{1}}{V}\right)^{a_{2}}\left(\frac{500}{\kappa_{2}}\right)^{0.266}-1\right] \\
& \times\left(\frac{V}{15}\right)^{a_{3}}\left(\frac{a_{4}}{\kappa_{1}}\right)^{0.7\left(\frac{\kappa_{1}}{0.1}\right)^{a_{5}}} ; \\
& a_{1}=8.899 \cdot 10^{-4} \kappa_{2}+1.611 ; \\
& a_{2}=-1.11 \cdot 10^{-4} \kappa_{2}+1.211 ; \\
& a_{3}=2.788 \cdot 10^{-5} \kappa_{2}+0.072 ; \\
& a_{4}=0.01 \quad \text { при } \quad \kappa_{1} \geq 0.005, \\
& a_{4}=-2.5 \kappa_{1}+0.0225 \quad \text { при } \kappa_{1}<0.005 ;
\end{aligned}
$$

$$
\begin{aligned}
& a_{5}=0.1 \quad \text { при } \kappa_{1} \geq 0.005, \\
& a_{5}=-40 \kappa_{1}+0.32 \quad \text { при } \kappa_{1}<0.005,
\end{aligned}
$$

которые верны с точностью не хуже 5\% в диапазоне изменения параметров $\kappa_{1}, \kappa_{2}: \kappa_{1} \geq 3 \cdot 10^{-3}$;

$$
\kappa_{2} \geq \kappa_{2 \min }\left(\kappa_{1}\right)=\frac{100}{1+0.8\left(\frac{\kappa_{1}}{0.2}\right)^{5}} .
$$

\section{Список литературы}

[1] Donnelly V.M., Kornblit A. // J. Vacuum Sci. Technol. A: Vacuum, Surfaces, and Films. 2013. Vol. 31. N 5. P. 050825.

[2] Sturges D.J., Oskam H.J. // J. Appl. Phys. 1964. Vol. 35. N 10. P. 2887-2894.

[3] Rubel M. et al. // J. Nucl. Mater. 1992. Vol. 196. P. 285-291.

[4] Дунаев В.В., Жиглинский А.Г., Сухомлинов В.С., Фабурина Э.Н. // ЖТФ. 1992. Т. 62. Вып. 2. С. 41-46.

[5] Raizer Y. Gas Discharge Physics. Berlin, NY.: Springer, 1991. P. 536.

[6] Chen X.P. // Phys. Plasma. 1998. Vol. 5. N 3. P. 804.

[7] Bohm D. // Characteristics of Electrical Discharges in Magnetic Fields. 1949. Vol. 13.

[8] Riemann K. // Plasma Sources Sci. Technol. 2009. Vol. 18. P. 014006.

[9] Benilov M. // The Plasma Sources Science and Technology. 2008. Vol. 18. N 1. P. 014005.

[10] Allen J. // Plasma Sources Sci. Technol. 2009. Vol. 18. P. 014004.

[11] Brinkmann R. // J. Phys. D: Appl. Phys. 2011. Vol. 44. N 4. P. 042002.

[12] Kino G., Shaw E. // Phys. Fluids. 1966. Vol. 9. N 3. P. 587-593.

[13] Chen F.F. Introduction to Plasma Physics. NY., London: Plenum Press, 1974.

[14] Tsankov T., Czarnetzki U. // Plasma Sources Science and Technology. 2017. Vol. 26. N 5. P. 055003.

[15] Kos L., Tskhakaya S., Jelic N. // Phys. Plasmas. 2015. Vol. 22. N 9. P. 093503.

[16] Riemann K. // J. Physics D: Appl. Phys. 2003. Vol. 36. N 22. P. 2811.

[17] Riemann K. // J. Physics D: Appl. Phys. 1991. Vol. 24. N 4. P. 493.

[18] Baalrud S., Hegna C. // Plasma Sources Science and Technology. 2011. Vol. 20. N 2. P. 025013.

[19] Riemann K. // Phys. Plasmas. 2006. Vol. 13. N 6. P. 063508.

[20] Godyak V. // Phys. Lett. A. 1982. Vol. 89. N 2. P. 80-81.

[21] Valentini $H . \quad / /$ Phys. Plasmas. 1996. Vol. 3. N 4. P. 1459-1461.

[22] Chen X. // Phys. Plasmas. 1998. Vol. 5. N 3. P. 804-807.

[23] Riemann K. // Phys. Fluids. 1981. Vol. 24. N 12. P. 2163-2172.

[24] Kaganovich I. // Phys. Plasmas. 2002. Vol. 9. N 11. P. 4788-4793.

[25] Raoul N. // IEEE Transactions on Plasma Sci. 2002. Vol. 30. N 1. P. 352-356.

[26] Godyak V., Sternberg N. // IEEE Transactions on Plasma Sci. 2003. Vol. 31. N 2. P. 303.

[27] Franklin R.N. // J. Phys. D: Appl. Phys. 2003. Vol. 36. N 22. P. 2821 . 
[28] Riemann K. // Plasma Sources Science and Technology. 2012. Vol. 21. N 6. P. 068001.

[29] Mustafaev A., Sukhomlinov V., Ainov M. // Tech. Phys. 2015. Vol. 60. P. 1778.

[30] Valentini H., Kaiser D. // Phys. Plasmas. 2015. Vol. 22. N 5. P. 053512.

[31] Liu J., Wang Z., Wang X. // Phys. Plasmas. 2003. Vol. 10. N 7. P. 3032-3034.

[32] Kotelnikov I., Skovorodin D. // Plasma Phys. Reports. 2016. Vol. 42. N 2. P. 186-190.

[33] Sukhomlinov V., Mustafaev A., Grabovskii A., Ainov M. Ion velocity distribution function in the plasma of its own gas // 42nd EPS Conf. on Plasma Physics. 2015. P5.168.

URL: http://ocs.ciemat.es/EPS2015PAP/pdf/P5.168.pdf

[34] Wang H., Sukhomlinov V., Kaganovich I., Mustafaev A. // Plasma Sources Sci. Technol. 2017. Vol. 26. N 2. P. 024001.

[35] Wang H., Sukhomlinov V., Kaganovich I., Mustafaev A. // Plasma Sources Sci. Technol. 2017. Vol. 26. N 2. P. 024002.

[36] Mustafaev A.S., Soukhomlinov V.S. // J. Mining Institute. 2016. Vol. 222. P. 864-868.

[37] Mustafaev A., Sukhomlinov V., Ainov M. // High Temperature. 2007. Vol. 55. N 3. P. $346-351$.

[38] Tonks L., Langmuir I.A // Phys. Rev. 1929. Vol. 34. N 6. P. 876.

[39] Sukhomlinov V.S., Mustafaev A.S., Murillo O. // Phys. Plasmas. 2018. Vol. 25. N 1. P. 013513.

[40] Maiorov S., Petrov O., Fortov V. Calculation of resonant charge exchange cross-sections of ions Rubidium, Cesium, Mercury and noble gases // Proceedings of the 34th EPS Conference on Plasma Physics, Warsaw. 2007.

URL: http://epsppd. epfl.ch/Warsaw/pdf2/P2_115.pdf

[41] Gill P., Webb C. // J. Phys. D: Appl. Phys. 1977. Vol. 10.

[42] Kolobov V.I., Tsendin L.D. // Plasma Sources Sci. Technol. 1995. Vol. 4. N 4. P. 551.

[43] Riemann K., Ehlemann U., Wiesemann K. // J. Phys. D: Appl. Phys. 1992. Vol. 25. N 4. P. 620.

[44] Riemann K. // Plasma Sourc. Sci. Technol. 2009. Vol. 18. P. 014007.

[45] Sheridan T. // Appl. Phys. Lett. 1996. Vol. 68. N 14. P. $1918-1920$.

[46] Sheridan T. // J. Phys. D: Appl. Phys. 2010. Vol. 43. N 10. P. 105204.

[47] Sheridan T. // Phys. Plasmas. 2000. Vol. 7. N 7. P. 3084-3088. 